\section{PO-0392 LATERALITY OF INTRAVENTRICULAR HAEMORRHAGE INFLUENCES NEURODEVELOPMENTAL OUTCOME IN PRETERM INFANTS}

${ }^{1} \mathrm{~K}$ Goeral, ${ }^{2} \mathrm{G}$ Kasprian, ${ }^{1} \mathrm{~L}$ Schmidt, ${ }^{1} \mathrm{~F}$ Cardona, ${ }^{1} \mathrm{~A}$ Berger, ${ }^{1} \mathrm{~K}$ Klebermass-Schrehof. ${ }^{1}$ Department of Pediatrics and Adolescent Medicine - Division of Neonatology Intensive Care and Pediatric Neurology, Medical University of Vienna, Vienna, Austria, ${ }^{2}$ Department of Radiology - Division of Neuroradiology and Musculoskeletal Radiology, Medical University of Vienna, Vienna, Austria

\subsection{6/archdischild-2014-307384.1038}

Background Intraventricular haemorrhage (IVH) is a significant cause of morbidity and mortality in premature infants. There is a well known correlation between IVH grade and neurodevelopmental outcome. However, to our knowledge, there are only a few studies taking into account the side of the lesion.

Methods Data of 178 infants with grade III IVH with and without parenchymal involvement were retrospectively analysed. Diagnosis was based on cerebral ultrasound. 36\% $(n=64)$ of neonates showed equal IVH severity on both brain sides, the remaining 64\% $(\mathrm{n}=114)$ had IVH severity which differed between the hemispheres (right $>$ left, $\mathrm{n}=53$; left $>$ right, $\mathrm{n}=61$ ).

Neurodevelopmental outcome was evaluated at 2 years corrected age via Bayley Scales of Infant development II (BSID II). The data was corrected for influence of gestational age by multiple regression analyses.

Results Mental Development Index (MDI) and Psychomotor Development Index (PDI) were compared between the groups and infants with a larger lesion within the right hemisphere showed statistically significant lower developmental scores at the age of two years (mean (median) \pm SD for 'right $>$ left' vs. 'left $>$ right': MDI 64,2 (54,0) \pm 19,6 vs. 78,8 (84,0) $\pm 17,6[\mathrm{p}=$ $0,047]$ and PDI $59,7(56,5) \pm 11,4$ vs. $75,9(80,0) \pm 17,1[\mathrm{p}=$ 0,021]).

Conclusion Laterality of IVH has a significant influence on neurodevelopmental outcome in preterm infants. Further studies referring to outcome at school age based on more sophisticated imaging techniques are warranted.

\section{PO-0393 RECOVERY OF OCCIPITAL-FRONTAL CIRCUMFERENCE Z-SCORE OF HEALTHY VERY PRETERM INFANTS AT TERM-EQUIVALENT AGE DOES NOT REFLECT A BRAIN SPARING EFFECT}

${ }^{1} \mathrm{R}$ Sousa, ${ }^{1} \mathrm{~K}$ Cardoso, ${ }^{2} \mathrm{AM}$ Graca. ${ }^{1}$ Department of Pediatrics, Lisbon Academic Medical Center, Lisboa, Portugal; ${ }^{2}$ NICU - Department of Pediatrics, Lisbon Academic Medical Center, Lisboa, Portugal

\subsection{6/archdischild-2014-307384.1039}

Background and aims To evaluate the growth of occipito-frontal circumference (OFC), length and weight, between birth and term equivalent age (TEA) of very preterm infants and to compare to somatometry of term-born control infants.

Methods We assessed prospectively a cohort of infants born at $\leq 32$ weeks gestation and term control newborns. Infants with cerebral abnormalities were excluded. Measurements of OFC, length and weight were performed at birth and TEA for preterm and at birth for term infants. Brain volume was estimated by a previously described ultrasonographic model (Graca A, Early Hum Dev 2013). Z-scores for somatometric variables were determined based on Fenton 2013 growth charts and z-scores for estimated brain volume were defined in controls. We used

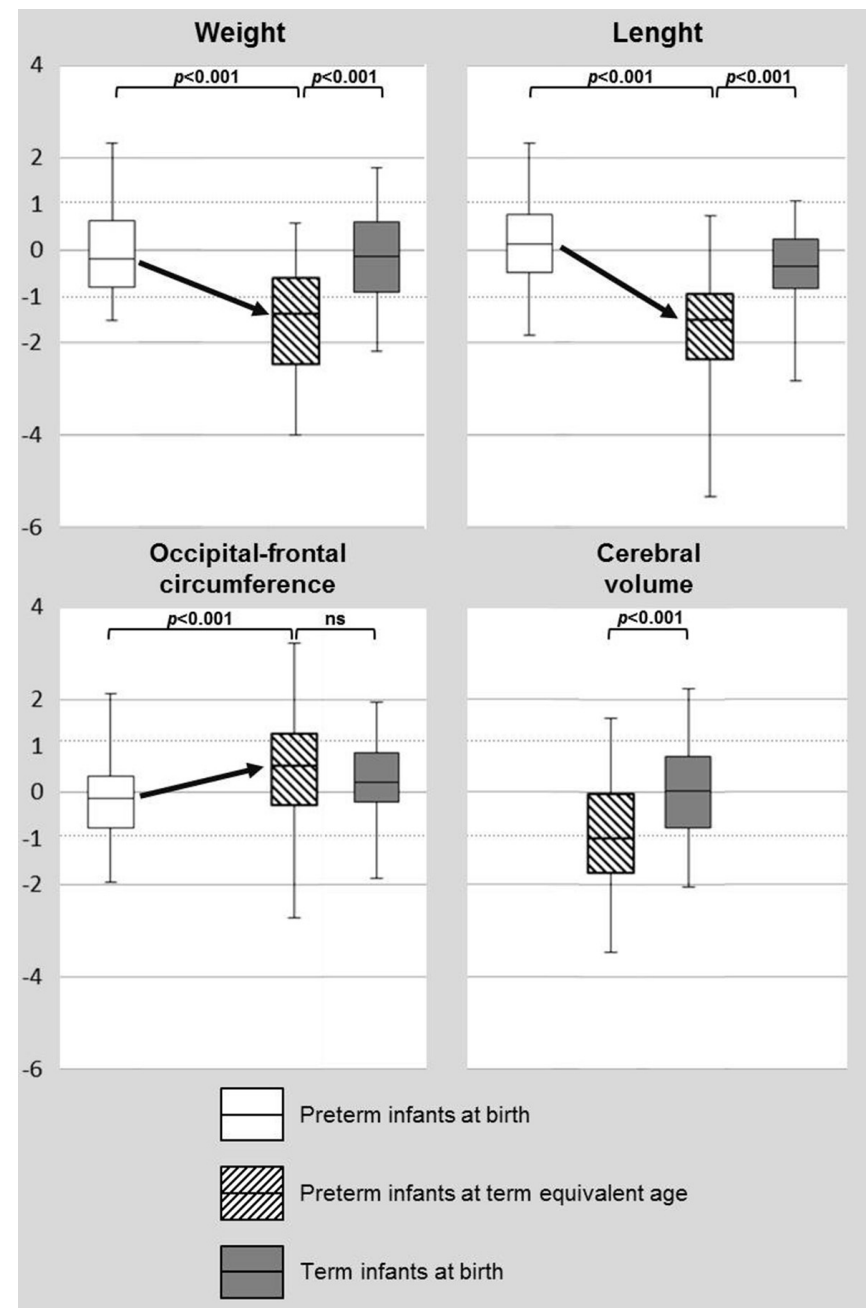

Abstract P0-0393 Figure 1

paired samples t-test to compare z-scores between birth and TEA for preterms and independent samples t-test to compare z-scores between preterm infants at TEA and controls.

Results We assessed 128 infants (72 preterms and 56 controls). Weight and length z-scores decreased significantly between birth and TEA and were significantly inferior to controls, whereas OFC $\mathrm{z}$-scores increased and were identical at TEA to controls (Figure). Estimated brain volume was nevertheless significantly inferior for preterm infants at TEA.

Conclusion Recovery of OFC z-score of healthy very preterm infants at TEA does not reflect a brain sparing effect, since despite OFC growing in a opposite direction than weight and length, estimated brain volume is significantly lower than controls.

\section{PO-0394 IS ACUTE KIDNEY INJURY RELATED TO WORSE OUTCOMES IN NEWBORNS TREATED WITH HYPOTHERMIA?}

${ }^{1} \mathrm{C}$ Gomes, ${ }^{1} \mathrm{~L}$ Oliveira, ${ }^{1} \mathrm{C}$ Gomes, ${ }^{2} \mathrm{~S}$ Sampaio, ${ }^{3} \mathrm{R}$ Stone, ${ }^{2} \mathrm{C}$ Moniz, ${ }^{2} \mathrm{AM}$ Graca. ${ }^{1}$ Department of Pediatrics, Lisbon Academic Medical Center, Lisboa, Portugal; ${ }^{2}$ NICU Department of Pediatrics, Lisbon Academic Medical Center, Lisboa, Portugal; ${ }^{3}$ Pediatric Nephrology Unit - Department of Pediatrics, Lisbon Academic Medical Center, Lisboa, Portugal

10.1136/archdischild-2014-307384.1040 
Background and aims Acute kidney injury (AKI) is a common consequence of perinatal asphyxia (PA). Therapeutic hypothermia $(\mathrm{TH})$ used for neuroprotection in hypoxic-ischaemic-encephalopathy (HIE) may also ameliorate kidney injury. AKI can be associated with more severe PA, but association with worse neurological outcome remains to be proven. We aim to evaluate the incidence of AKI in neonates under $\mathrm{TH}$ and the association with HIE grades and outcome.

Methods A total of 51 cooled infants were reviewed using a prospectively collected database. Modified AKIN criteria were used to classify AKI. We studied perinatal, resuscitation and clinical data during admission to the NICU. Outcome of interest was duration of NICU stay, predictable neurologic outcome and mortality.

Results AKI was found in 17 of 51 neonates (33\%). Based on the modified AKIN criteria, 13, 1, and 3 patients had stages I, II, and III, respectively. AKI was more frequent in HIE $2 / 3$ than in the rare HIE 1 infants that were cooled $(\mathrm{p}<0.03)$ and in those with worse base deficit at birth $(p<0.05)$. A significant association was found between vancomycin and gentamicin use and AKI $(\mathrm{p}<0.01)$. Renal replacement therapy was needed in $2 / 3$ of AKIN stage III infants. NICU stay, mortality and predicted adverse neurologic outcomes were not associated with AKI.

Conclusions AKI was less frequent in our cohort than the one previously described in non-cooled newborns. More severely asphyxiated neonates were more likely to experience AKI, but AKI was not related to worse outcomes.

\section{PO-0395 SCAVENGING OF EXTRACELLULAR HAEMOGLOBIN MODIFIES THE MONOCYTE-MACROPHAGE RECRUITMENT AND DIFFERENTIATION IN THE INTRAVENTRICULAR SPACE FOLLOWING IVH}

${ }^{1} \mathrm{M}$ Gram, ${ }^{1} \mathrm{~S}$ Sveinsdottir, ${ }^{1} \mathrm{~S}$ Vallius, ${ }^{1} \mathrm{~K}$ Sveinsdottir, ${ }^{2} \mathrm{M}$ Cinthio, ${ }^{1} \mathrm{D}$ Ley. ${ }^{1}$ Pediatrics, Lund University, Lund, Sweden; ${ }^{2}$ Electrical Measurments, Lund University, Lund, Sweden

10.1136/archdischild-2014-307384.1041

Introduction Severe cerebral intraventricular haemorrhage (IVH) in preterm infants continues to be a major clinical problem. To date, no available therapy prevents infants from neurologic sequel following IVH. Recruitment of monocytes-macrophages and periventricular infiltration is a key step in the inflammatory response leading to brain damage. The sequence of the recruitment and profiling of monocytes-macrophages following IVH is not well characterised. We have previously shown that extracellular haemoglobin induces chemotactic cytokines following IVH. Haptoglobin is a haemoglobin scavenger and could potentially protect the immature brain from the detrimental effects of haemoglobin.

Objective To characterise the recruitment and differentiation of monocytes-macrophages in the intraventricular space following IVH and to investigate if haemoglobin scavenging with haptoglobin alters the recruitment and differentiation.

Methods Using a preterm rabbit pup model of IVH we characterised the immune cell recruitment and differentiation in intraventricular cerebrospinal fluid (CSF) at 24 to $72 \mathrm{~h}$ following haemorrhage. Using flow cytometry, immunohistochemistry and mRNA and protein analysis we characterised the systemic and CSF infiltrating macrophages in animals with IVH, sham controls and animals treated with intraventricular injections of haptoglobin.

Results Following IVH, there is an infiltration of M1 macrophages into the intraventricular CSF. Intraventricular introduction of the haemoglobin-scavenger haptoglobin modifies them into alternative M2 macrophages, expressing CD163. This causes a subsequent in vivo clearance of the accumulated haemoglobin.

Conclusion Following IVH, intraventricular haptoglobin treatment modifies macrophage differentiation, initiating clearance of extracellular haemoglobin. Treatment of haptoglobin might be a feasible approach to protect the immature brain following IVH.

\section{P0-0396 THE SIGMA-1 RECEPTOR AGONIST PRE-084 PROTECTS AGAINST GLUTAMATE INDUCED NEUROTOXICITY IN PRIMARY HIPPOCAMPAL NEURONS}

${ }^{1}$ E Griesmaier, ${ }^{1} \mathrm{M}$ Urbanek, ${ }^{1} \mathrm{~K}$ Stock, ${ }^{2} \mathrm{M}$ Hermann, ${ }^{1} \mathrm{~A}$ Posod, ${ }^{3} \mathrm{RI}$ Stanika, ${ }^{3} \mathrm{GJ}$ Obermair, ${ }^{1} \mathrm{U}$ Kiechl-Kohlendorfer. 'Paediatrics II Neonatology, Innsbruck Medical University, Innsbruck, Austria; ${ }^{2}$ Anaesthesiology and Critical Care Medicine, Innsbruck Medical University, Innsbruck, Austria; ${ }^{3}$ Physiology and Medical Physics, Innsbruck Medical University, Innsbruck, Austria

\subsection{6/archdischild-2014-307384.1042}

Background Prematurity is a major determinant of neonatal mortality and morbidity. The number of preterm birth is still on the rise. Recently we and others could demonstrate neuroprotective effects of sigma-1 receptor ligands in adult and newborn animal models of brain injury. Since sigma-1 receptor agonists are already undergoing clinical trials in adult neurological disease, they might be considered a promising therapeutic option also in preterm brain injury. We have previously shown that the selective sigma-1 receptor agonist PRE-084 (2-(4-morpholinethyl)1-phenylcyclohexane-carboxylate) protects against neonatal excitotoxic brain injury in vivo. The aim of the present study was to investigate whether PRE-084 is able to prevent neurotoxicity following glutamate exposure in vitro.

Methods Cultured primary hippocampal neurons (day in vitro 10) were pre-treated with PRE-084 before glutamate was applied. Subsequently cell death was quantified by means of PI/ calcein - AM staining using live confocal microscopy. Neurons were randomly assigned to one of the following groups: i) control, ii) glutamate or iii) glutamate+PRE-084. PRE-084 was applied in two dosages $(10$ and $100 \mu \mathrm{M})$ prior to glutamate.

Results The application of PRE-084 significantly reduced the percentage of dead cells (PRE-084 $10 \mu \mathrm{M}$ : 22.09 (20.50;28.84) and $100 \mu \mathrm{M}$ : $25.87(18.77 ; 33.40))$ compared to the untreated glutamate control group $43.56(39,86 ; 46.02)$.

Conclusion Our data show that administration of PRE-084 protects against glutamate induced cell death in primary hippocampal neurons. PRE-084 shows considerable promise as a therapeutic strategy in preterm brain injury and might provide an adequate means of combating this major cause of neurological disability in infancy.

\section{P0-0397 THE NEUROPEPTIDE SECRETONEURIN IS PROTECTIVE IN ESTABLISHED IN VIVO AND IN VITRO MODELS OF NEONATAL BRAIN INJURY}

${ }^{1} \mathrm{E}$ Griesmaier, ${ }^{1} \mathrm{~K}$ Wechselberger, ${ }^{1} \mathrm{~A}$ Posod, ${ }^{1} \mathrm{~K}$ Stock, ${ }^{2} \mathrm{RI}$ Stanika, ${ }^{2} \mathrm{GJ}$ Obermair, ${ }^{3} \mathrm{M}$ Hermann, ${ }^{1} \mathrm{U}$ Kiechl-Kohlendorfer, ${ }^{1} \mathrm{M}$ Urbanek. ${ }^{1}$ Department of Paediatrics II Neonatology, Innsbruck Medical University, Innsbruck, Austria; ${ }^{2}$ Department of Physiology and Medical Physics, Innsbruck Medical University, Innsbruck, Austria; ${ }^{3}$ Department of Anaesthesiology and Critical Care Medicine, Innsbruck Medical University, Innsbruck, Austria

10.1136/archdischild-2014-307384.1043 